\title{
Effects of Glucose Restriction on Replicative Senescence of Human Diploid Fibroblasts IMR-90
}

\author{
Junhua Jin Tiemei Zhang \\ The Key Laboratory of Geriatrics, Beijing Hospital \& Beijing Institute of Geriatrics, Ministry of Health, \\ China
}

\author{
Key Words \\ Glucose restriction $\cdot$ Replicative senescence $\cdot$ Cell proliferation
}

\begin{abstract}
Background/Aims:. The aim of this study was to elucidate the effects of glucose restriction (GR) on cell replicative senescence in vitro by human diploid fibroblasts IMR-90. Methods: IMR-90 cells were cultured under $40,60 \%$ GR or high glucose medium and biomarkers of cell senescence were compared with cells cultured in normal glucose medium (5.5 mM glucose). The impact of different concentrations of glucose and initial passages on cell replicative senescence were assessed by cell survival days, cumulative population doublings (PD), cell proliferation rate (CPR) and SA- $\beta$-gal site-stain. Results: When compared with control cells, mean survival days and lifespan of IMR-90 were increased $16.7 \%$ and $11.4 \%$ by $40 \%$ GR $(3.3$ $\mathrm{mM}$ glucose). However, mean survival days and lifespan of IMR-90 were decreased $31.0 \%$ and $26.9 \%$ by $\mathrm{HG}$ treatment ( $25.0 \mathrm{mM}$ glucose). The effects on survival days of IMR-90 were associated not only with different glucose concentrations but also with initial passages. The CPR of IMR-90 could be retarded by GR culture and this effect was especially associated with GR degree. It was $87 \%$ positive cells of SA- $\beta$-gal in aging stages and more slim and fibrous cells were observed in 40\% GR group than NG group onset from 26 PD. Conclusion: Mean survival days and lifespan of human diploid fibroblasts IMR-90 were extended by glucose restriction. The higher GR levels, the earlier onset of GR, the larger benefits on extending survival days of IMR-90 could be observed. Slowing down cell proliferation by GR increased the number of cell survival days, an effect associated with GR levels. High glucose induced premature senescence of IMR-90 when started from any passages.
\end{abstract}

Copyright (C) 2013 S. Karger AG, Basel

Dr. Tiemei Zhang

The Key Laboratory of Geriatrics, Beijing Hospital \& Beijing Institute of Geriatrics Ministry of Health, Beijing Dongdan Dahua road No.1, Beijing 100730 (China)

Tel. +8610-58115044, Fax +8610-65237929, E-Mail tmzhang126@126.com 


\section{Introduction}

CR is by far the most effective environmental manipulation to extend maximum or mean lifespan in many different species [1-3]. Many studies found not only the longevity actions of CR but also a number of important CR anti-aging effects. CR has been shown to delay a wide range of aging-associated diseases, such as cancer, diabetes, atherosclerosis, cardiovascular diseases and neurodegenerative diseases in higher mammals, such as nonhuman primates and humans [4-6]. CR animals show especially a reduction in tumor development, decreasing progressively the proliferative potential of cells with age $[7,8]$. However, when and what degrees of CR for human can obtain the greatest benefits on antiaging or health life? Animal and human models could not give the last answer so far [2-10]. And the studies of assessing the possible impacts of long-term CR (>2years) on human are very difficult $[9,10]$.

An in vitro cellular system for CR has more advantages such as flexible control, accuracy and identical genomic background as compared to the in vivo systems. Human diploid fibroblast IMR-90 is one of cell lines commonly and widely used in aging research [11-13]. Previously, we established an in vitro system of IMR-90 to mimic CR-controlled longevity by glucose restriction (GR), the main caloric resource in cell culture medium. The mean lifespan of IMR-90 was significantly increased by $40 \%$ GR treatment compared with the normal control in our previous study [13]. The purpose of this study tries to elucidate the detail effects of glucose restriction on cells growth and senescence of human diploid fibroblasts IMR-90. This study may evaluate more details and insights for CR study in human and a mimic experimental system to investigate the molecular mechanisms by which CR mediates its biologic effects on cellular level.

\section{Materials and Methods}

\section{Cell culture}

Human diploid fibroblast cell line IMR-90 from American Type Culture Collection (ATCC CCL-186,USA) was normally cultured using Dulbecco's Modified Eagle Medium (5.5 mM D- glucose, Gibco) supplemented with $3.7 \mathrm{~g} / \mathrm{L}$ sodium bicarbonate, $2.0 \mathrm{mM}$ glutamine, $100 \mathrm{U} / \mathrm{ml}$ penicillin sodium, $100 \mathrm{U} / \mathrm{ml}$ streptomycin and $10 \%$ fetal calf serum (Front Biomedicine Company). The cells were seeded at a density of $2 \times 10^{4}$ cells / $\mathrm{cm}^{2}$. After seeding, the cells were allowed to attach, grow, and proliferate in an incubator at $37^{\circ} \mathrm{C}$ in a humid environment with $5 \% \mathrm{CO}_{2}$. Culture medium was changed every 2 days. To quantify replication, cells were digested by $0.25 \%$ trypsin $+0.3 \%$ EDTA solution until cells reached $90-95 \%$ confluence, counted number using a haemocytometer. The number of doublings for each passage was calculated from the formula $\mathrm{N}=$ $3.32\left(\log \mathrm{N}_{2}-\log \mathrm{N}_{1}\right)+\mathrm{X}$, where $\mathrm{N}$ is the final cells population doublings (PD), $\mathrm{N}_{2}$ is the final number of cells after the passage, $\mathrm{N}_{1}$ is the initial number of viable cells seeded and $\mathrm{X}$ is the previous cells PD (formula provided by ATCC).

\section{Cell groups}

Cell growth experiments were observed and recorded accurately and systematically on population doublings (PD), survival days, cell proliferation rate of IMR-90 cultured under NG, GR and HG conditions, respectively. The groups of IMR-90 with different concentrations of glucose treatment and initial PD were represented as Table 1. To reduce glucose, IMR-90 were cultured in glucose-free DMEM (Sigma-Aldrich) supplemented with different concentration of D-glucose (Sigma-Aldrich) and 10\% fetal bovine serum (HyClone). In this study, we provide 2.2, 3.3, 5.5 and $25.0 \mathrm{mM} \mathrm{D-glucose}$ to serve as $60 \% \mathrm{GR}, 40 \% \mathrm{GR}, \mathrm{NG}$ and HG group, respectively (Table 1). Each group had 3 parallel points and repeated at least twice and was subjected to statistical analysis.

Survival days and lifespan of IMR-90

Human diploid fibroblasts have a limited ability to proliferate under normal culture, a property known as the Hayflick limit [14]. In this state of growth arrest, referred to as replicative senescence, cells 
Table 1. Groups of IMR-90 cultured under medium with different concentrations of glucose and initial PD

\begin{tabular}{lcccc}
\hline & NG & LG2.2 & LG3.3 & HG \\
\hline Glucose conc. (mM) & 5.5 & 2.2 & 3.3 & 25.0 \\
GR level (\%) & 0 & 60 & 40 & \\
& 26 & & 26 & 26 \\
& 30 & 30 & 30 & 30 \\
Initial PD & 35 & 35 & 35 & 35 \\
& 40 & 40 & 40 & 40 \\
& 45 & 45 & 45 & 45 \\
\hline
\end{tabular}

Table 2. The average cell proliferation rate (days/PD) of IMR90 was shown during different stages when cells were cultured under NG condition and initiated from different passages

\begin{tabular}{lcccc}
\hline Initial PD & $\begin{array}{c}30 \\
(\mathrm{n}=5)\end{array}$ & $\begin{array}{c}35 \\
(\mathrm{n}=5)\end{array}$ & $\begin{array}{c}40 \\
(\mathrm{n}=6)\end{array}$ & $\begin{array}{c}45 \\
(\mathrm{n}=5)\end{array}$ \\
Stage of cells & Average cell proliferation rate (Days/PD) \\
\hline 30-35PD & $2.9 \pm 1.2$ & & & \\
36-40PD & $3.5 \pm 1.0$ & $3.3 \pm 0.9$ & & \\
41-45PD & $4.3 \pm 0.8$ & $4.0 \pm 0.9$ & $3.6 \pm 0.9$ & \\
46-50PD & $5.0 \pm 0.8$ & $4.7 \pm 1.0$ & $4.4 \pm 0.7$ & $5.1 \pm 1.4$ \\
51-55PD & $7.5 \pm 2.2$ & $7.3 \pm 3.1$ & $8.2 \pm 3.1$ & $8.8 \pm 2.4$ \\
\hline
\end{tabular}

adopt a typical large and flat morphology and express a number of senescence-associated (SA) markers. The lifespan of IMR-90 populations cultured under normal condition is about 50-60 PD and it would take 3-5 months (survival days) to reach growth arrest. The whole cell growth shows a steady growth between 20 and $40 \mathrm{PD}$ at first, then slowing growth between 40 and $50 \mathrm{PD}$, and finally, population senescence between 50 and 60 PD $[11,13,15]$. In this study, IMR-90 took about 150 days to reach growth arrest at about 55 PD passages onset from 26 PD, similar as Nichols reported [15]. Cell culture experiments were terminated after 2 weeks when cells stop proliferation and reach the growth arrest. The deadline of cell culture days was defined as survival days of IMR-90. The initial PD was designated as PD 0. The cumulative PD of last passages was defined as lifespan of IMR-90 in this study.

\section{Cell proliferation rate}

Cell proliferation rate (CPR) was defined as the subculture days of every passage (days/PD) in this study. Under NG condition, the average CPR of every 5 PD during steady growth (20-40 PD) is about 3-4 days/PD, during slowing growth (40-50 PD) is about 4-5 days/PD and senescence stage ( $\geq 50$ PD) is about 7-8 days/PD, respectively (Table 2). No significant difference was shown between the same stages under NG condition onset from different PD. There is a relative stable CPR of IMR-90 in NG medium during different growth stages. These data of CPR under NG condition were consistent with human diploid fibroblasts growth characteristics in other studies $[11,13,15]$.

\section{Senescence-associated $\beta$-galactosidase (SA- $\beta$-gal) activity assay}

SA- $\beta$-gal stain solution was included $5 \mathrm{mM} \mathrm{K}_{4} \mathrm{Fe}(\mathrm{CN})_{6}, 5 \mathrm{mM} \mathrm{K}_{3} \mathrm{Fe}(\mathrm{CN})_{6}, 2 \mathrm{mM} \mathrm{MgCl}, 40 \mathrm{mM}$ sodium citrate ( $\mathrm{pH} 6.0$ ) prepared by our laboratory. $10^{5}$ cells/well were seeded in 24 -well plate and after completely adherent to the plate, the cells were washed with phosphate-buffered saline (PBS) twice, fixed in $3 \%$ formaldehyde at room temperature for $15 \mathrm{~min}$ and then wash with PBS three times. Cells were incubated at $37{ }^{\circ} \mathrm{C}$ overnight with fresh SA- $\beta$-gal stain solution containing $1 \mathrm{mg} / \mathrm{ml}$ of 5 -bromo-4-chloro-3-indolylbeta-D-galactoside (X-gal, Amresco product). The old cell samples using as SA- $\beta$-gal activity tests in $5.5,2.2$ and 3.3 mM G groups were about 50-53 PD passages. The cell samples in HG groups were 41-43 PD started from 26 and 30 PD, 43-45 PD started from 35 PD, 45-47 PD started from 40 PD, and 46-48 PD started from $45 \mathrm{PD}$, respectively. The SA- $\beta$-gal positive cells with dark green color were taken pictures by using a Kodak DC120 Zoom digital camera (Kodak, Japan) at 40-fold magnification. Each group had 3 parallel wells and every test was repeated 3 times at least.

\section{Statistical analyses}

Data shown in this article are means $\pm S D$ from at least three different determinations. Significant differences between means were assessed using Student's $t$ test. Differences were considered significant with $p<0.05$. Data were analyzed using Sigma plot 10.0 Software. 
Fig. 1. Glucose restriction extends survival days and lifespan of human fibroblasts. Mean survival days of IMR-90 were extended about 30 days by $3.3 \mathrm{mmol} / \mathrm{L}$ glucose and reduced about 53 days by $25.0 \mathrm{mmol} / \mathrm{L}$ glucose (A). Cumulative population doublings was increased 3-4 PD by $3.3 \mathrm{mmol} / \mathrm{L}$ glucose and decreased 8-9 PD by $25.0 \mathrm{mmol} / \mathrm{L}$ glucose (B). A positive linear correlation between the extents of survival days relative to control and GR levels was shown in C. Experiments were terminated when cells stopped proliferating and underwent replicative senescence in 2 weeks. Population doublings were calculated by the formula ([Cnumber of cells harvested) /(number of cells seeded)] / log2, initial PD set to zero). Each data depicts the averaged results from three longevity assessments. $\left({ }^{* *} \mathrm{p}<0.01\right.$ vs NG or HG).

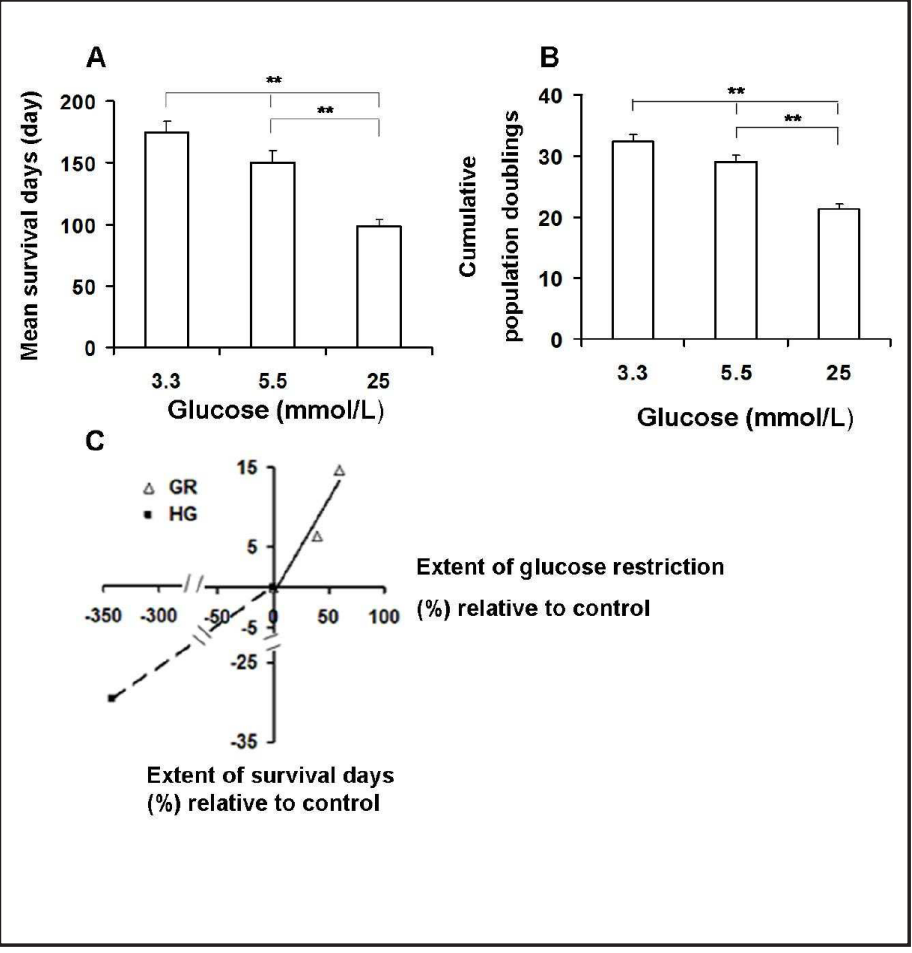

\section{Results}

Glucose restriction impacts on survival days and lifespan of IMR-90

Our previous study found that glucose restriction (GR) culture could increase the lifespan of IMR-90 fibroblasts compared with cells in normal culture condition [13]. It's necessary to verify whether this retarding aging could be induced by other levels of GR and what time to initial GR culture could receive the benefit on retarding replicative senescence of IMR-90. We therefore extended this study to develop a cell model to further assess the role of different glucose restriction levels on cell replicative senescence in vitro.

As indicated in Fig. 1A and B, mean survival days of IMR-90 were $150.0 \pm 10.0$ days and the cumulative populations were 29.0 \pm 1.1 PD when cells grown in NG medium (onset from 26 PD). Mean survival days were extended by 25-34 days accompanied by an additional 3-4 PD, which constituted $16.7 \%$ increase on survival days and $11.4 \%$ prolongation of lifespan when cells grown in $40 \%$ GR medium ( $3.3 \mathrm{mM}$ ). However, mean survival days were reduced by 50-55 days accompanied by a minus $8-9$ PD, which decreased $31.0 \%$ on survival days and $26.9 \%$ on lifespan when cells grown in HG medium $(25.0 \mathrm{mM})$. These results show that survival days and lifespan of IMR-90 could be extended under the situations of GR or caloric restriction (CR) and HG could induce premature senescence. Consistent with our previous studies, these results further established that CR can induce longevity not only in experimental animal models, but also in a culture cellular system $[11,13]$.

The role of GR levels on survival days of IMR-90

In order to further assess the role of GR levels on longevity of IMR-90, mean survival days were observed when IMR-90 were treated with different concentrations of glucose as $60 \%$ and $40 \%$ GR groups relative to control onset from 30 PD. Mean survival days of IMR-90 were extended $14.7 \%$ by $60 \%$ GR and $6.4 \%$ by $40 \%$ GR, respectively. No effect on replicative senescence could be seen by low level of $20 \%$ GR ( $4.4 \mathrm{mM}$, data not show). The relationship among the levels of GR and the extents of survival days increased was shown in Fig.1C. The 


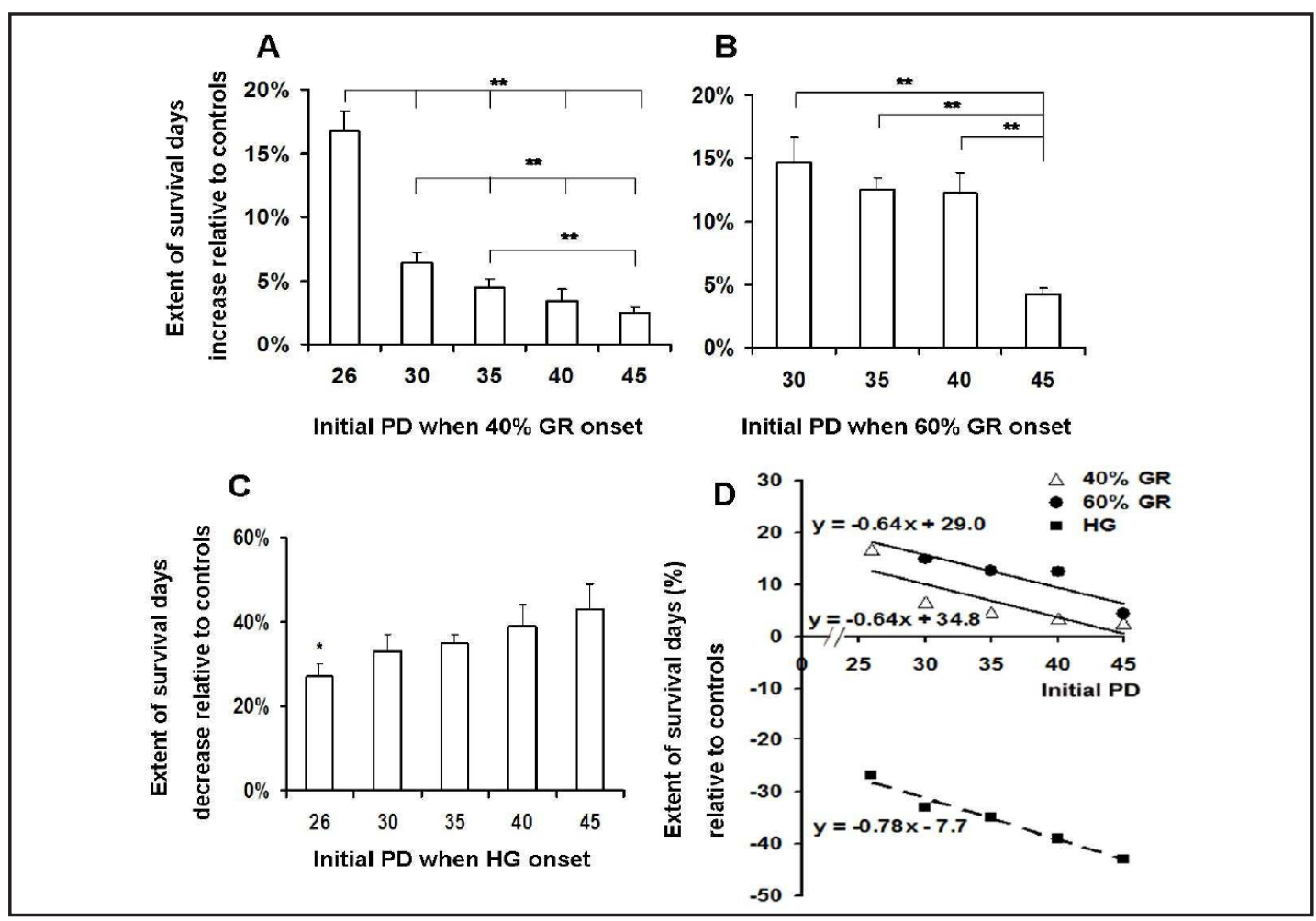

Fig. 2. The effect of initial PD on relative benefit of survival days of IMR- 90 was shown in different glucose concentrations. Mean survival days were increased $16.7 \%, 6.4 \%, 4.4 \%, 3.4 \%$ and $2.5 \%$ relative to controls when IMR-90 started from 26, 30, 35, 40 and 45 PD under 40\% GR medium, respectively (A). Mean survival days were increased 14.7\%, 12.6\%, 12.3\% and 4.2\% relative to controls when IMR-90 started from 30, 35, 40 and 45 PD under 60\% GR medium, respectively (B). Mean survival days were decreased 26.9\%, 32.8\%, $35.4 \%, 39.3 \%$ and $42.9 \%$ relative to controls when IMR-90 started from 26, 30, 35, 40 and 45 PD under HG medium, respectively (C). The parallel linear relationship of the extents of survival days and initial PD by $40 \%, 60 \%$ GR and HG was shown in D. (* $\left.\mathrm{p}<0.05,{ }^{* *} \mathrm{p}<0.01\right)$.

data show that there is a strong linear correlation between the degree of GR and the extents of survival days increased relative to controls. High glucose $(25.0 \mathrm{mM})$ was set as opposite group ( $-350 \%$ GR relative to NG) and there is a linear correlation between HG and the extent of survival days decreased (Fig.1C).

The effect of initial passages on survival days of IMR-90

Most animal studies are weaning-initiated caloric restriction, despite of fact that adultinitiated caloric restriction might be a feasible option for human use. It is very important to verify accuracy what time for CR onset to acquire the greater benefits on anti-aging. In order to observe the effect of different initial passages on cellular senescence, IMR-90 was started from 26, 30, 35, 40 and 45 PD under GR, NG or HG medium, respectively. Mean survival days of IMR-90 were $150.0 \pm 10.0,130.8 \pm 8.9,113.0 \pm 11.8,88.0 \pm 5.9$ and $71.4 \pm 6.5$ days in NG medium onset from $26,30,35,40$ and $45 \mathrm{PD}$, respectively.

Firstly, mean survival days of IMR-90 in 40\% GR medium were carefully observed when IMR-90 initiated from different passages. When IMR-90 was started from 26, 30, 35,40 and 45 PD, mean survival days were extended by 25.0, 8.4, 5.0, 3.0 and 1.8 days which increased $16.7 \%, 6.4 \%, 4.4 \%, 3.4 \%$ and $2.5 \%$ relative to controls, respectively (Fig. $2 \mathrm{~A}$ ). These results show that it is never too late to start GR culture on extending survival days of IMR-90. However, the earlier to start GR culture, the larger benefit could be acquired on retarding replicative senescence of IMR-90. And the benefit on retarding replicative 
Table 3. The ratio of $60 \% / 40 \%$ GR of relative extent of increased survival days when IMR-90 was initiated from different passages

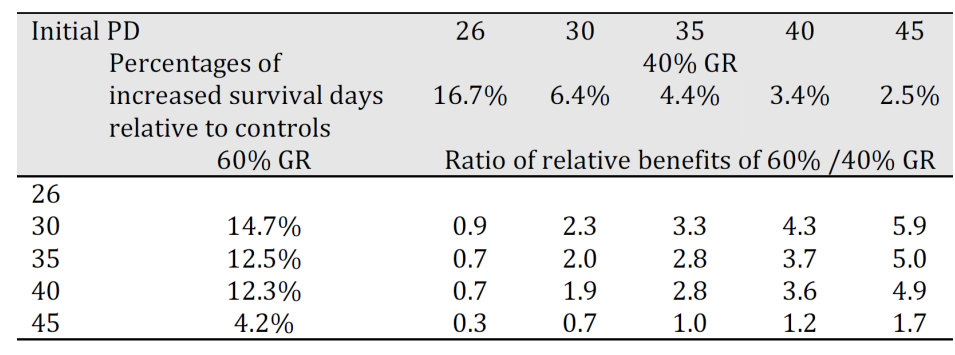

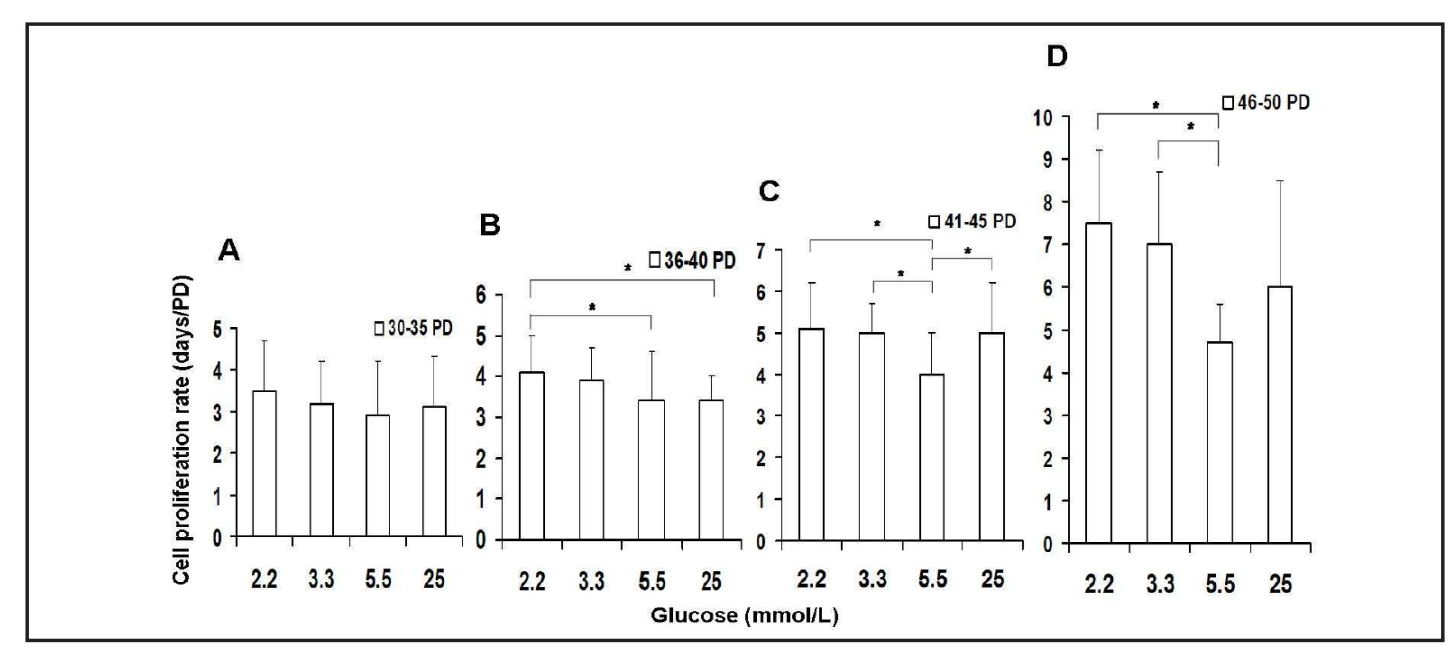

Fig. 3.The average of cell proliferation rate (subculture days/PD) of IMR-90 was slow down by GR. The average CPR of every 5 passages was observed when IMR-90 cultured under 2.2, 3.3, 5.5 and $25.0 \mathrm{mM}$ glucose medium, respectively. No significant difference of CPR was found among groups during the first 5-PD stage (A). The average CPR was significantly slow down by $60 \%$ GR treatment during the second 5-PD stage (B). The average CPR was significantly retarded by both 40 and $60 \%$ GR treatments during the third 5-PD stage (C) and maintained this effect until to old stage (D). $(* \mathrm{p}<0.05)$.

senescence of IMR-90 could be declined rapidly by $40 \%$ GR as initial passages addition. This pattern of declining benefit of GR is consistent with CR animal that the later the restriction start, the lower the benefit is acquired on lifespan extension [2,16-18].

Secondly, in order to further verify the effect of initial passage on survival days of IMR90 by other GR levels, cells were cultured in 60\% GR medium and started from different passages. When IMR-90 was started from 30, 35, 40 and 45 PD in 60\% GR medium, the mean survival days were extended by $19.2,14.2,10.8$ and 3.0 days which increase $14.7 \%, 12.6 \%$, $12.3 \%$ and $4.2 \%$ relative to controls, respectively (Fig. 2B). There were similar results as above that it's never too late to start GR culture on extending survival days of IMR-90. These results further confirm the fact that the earlier to start GR culture, the larger benefit could be acquired on retarding replicative senescence of IMR-90.

But things were slightly changed when IMR-90 was cultured in $60 \%$ GR medium. The pattern of declining impact of higher GR level is different to lower GR level. The data show that there were kept above $10 \%$ increase of survival days by $60 \%$ GR onset from stable growth (30-40 PD). However, only when started from early stage (26 PD), above $10 \%$ increase of survival days could be acquired by $40 \%$ GR. And the relative benefits by $60 \%$ GR onset from old cells were similar as the average percentages of relative benefits by $40 \%$ GR onset from stable growth (30-40 PD).

Further to verify the relationship between the impacts of GR levels and initial passages, the ratios of $60 \% / 40 \%$ GR of relative benefit of survival days increase among different initiated groups were shown in Table 3. Compared with these ratios, it's very easy to find that the relative benefit of $60 \%$ GR onset from stable growth (30-40 PD) was similar as $40 \%$ GR from early(26 PD, ratio=0.7-0.9). And the relative benefit of $60 \%$ GR onset from old 
Fig. 4. SA- $\beta$-gal activity of IMR-90 under GR or HG treatment. Old cells SA- $\beta$-gal activity of IMR-90 cultured in different concentrations of glucose (5.5, 2.2, 3.3 and $25.0 \mathrm{mmol} / \mathrm{L})$ was shown when started from 26 PD (A), 30 PD (B), 35 PD (C), 40 PD (D) and 45 $\mathrm{PD}(\mathrm{E})$, respectively. The magnification was $40 \times$. It was $89 \%, 96 \%$ and $98 \%$ positive aging cells of SA- $\beta$-gal under $40 \%$ GR, NG and HG onset from $26 \mathrm{PD}$, respectively (A). Although there was no significantly difference of SA- $\beta$-gal positive cells in old stages by $40 \%$ and $60 \%$ GR treatments, much more old cells were kept slim as fibrous in morphology by $40 \%$ and $60 \%$ GR versus NG or HG, respectively (B, C, D).

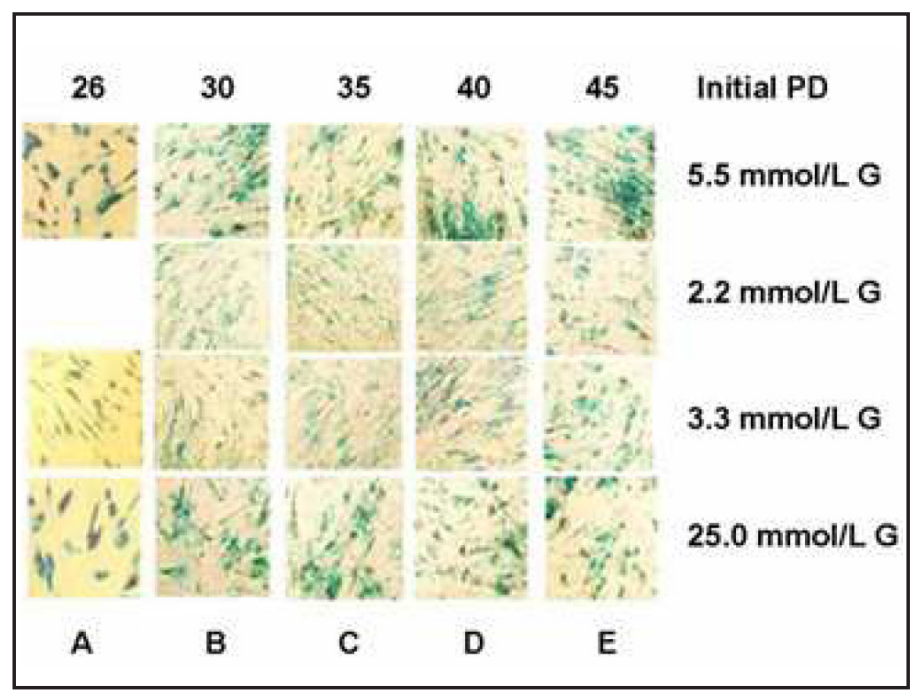

cells (45 PD) was similar as $40 \%$ GR from stable growth (30-40 PD, ratio=0.7-1.2). But the relative benefits of $60 \%$ GR were larger than $40 \%$ GR onset from same stable stages (30-40 $\mathrm{PD}$, ratio=1.9-5.9).

Thirdly, the impacts of initial passages on survival days by HG treatment were also observed. When IMR-90 was cultured and started from 26, 30, 35, 40 and 45 PD under HG medium, the mean survival days were decreased $26.9 \%, 32.8 \%, 35.4 \%, 39.3 \%$ and $42.9 \%$ relative to controls, respectively (Fig. 2C). These results show that HG treatment could induce premature senescence of IMR-90 initial from any passages and the extents of survival days were deceased greater when IMR-90 initiated from older cells.

In order to observe intuitive relationship between initial PD and the effects of GR or HG impacts on longevity, the trend lines of initial PD and the effects of GR or HG on survival days were drawn into Fig. 2D. A nearly parallel linear correlation was shown among the trend lines of initial PD and the extents of survival days relative to controls (Fig. 2D). From these lines, it also shows that the higher degree of GR, the earlier onset of GR, the larger benefits on retarding replicative senescence could be acquired. And the later onset of $\mathrm{HG}$, the larger effects on inducing premature senescence could be received.

\section{Cell proliferation rate retarded by GR treatment}

Average of every 5 passages of cell proliferation rate (CPR) was monitored very accurately during the entire growth of IMR-90. During the first 5-PD stage (30-35 PD), no significant difference of average CPR was observed among NG, 40\%, 60\% GR and HG groups (Fig. 3A). From the second 5-PD stage (36-40 PD), the average CPR in 60\% GR group was significantly slow than NG and HG groups and this impact has been maintained throughout the entire growth (Fig. 3B, C and D). From the third 5-PD stage (41-45 PD), the average CPR in $40 \%$ GR group became slow than NG group and this impact was also kept to old stage (Fig. $3 \mathrm{C}$ and $\mathrm{D}$ ). Although the average CPR in HG group also became slow than NG group during the third 5-PD stage, it's not maintained to old stage (Fig. 3C and D). These data show that only after 5 passages subculture by $60 \%$ GR treatment, the average CPR could be retarded more slowly than normal cells and this effect could have been maintained during the whole growth until to cell senescence. But it should be after 10 passages subculture to have the slowing impact of CPR by $40 \% \mathrm{GR}$.

\section{SA- $\beta$-gal activity of IMR-90 under GR treatment}

To determine the effect of GR or HG on replicative or premature senescence, we observed cells in their aging stages under the condition of either GR or HG medium by using senescence-associated $\beta$-galactosidase (SA- $\beta$-gal) activity assay. We found that IMR-90 under 
NG, GR or HG medium show a very low proportion of positive cells with SA- $\beta$-gal stain during steady growth stages. Further, no prominent differences in SA- $\beta$-gal activity were observed among cells with the treatment of NG, GR or HG medium in steady stages started (data not shown). Successive subculture resulted in increased positive senescent cells of SA- $\beta$-gal accumulation in aging cells (Fig. 4). It was $89 \%, 96 \%$ and $98 \%$ positive aging cells of SA- $\beta$ gal under 40\% GR, NG and HG medium onset from 26 PD, respectively (Fig. 4A). This result indicates that cells in aging stages by $40 \%$ GR treatment were kept slim as fibrous more than NG and HG groups. Most cells in aging stages by NG or HG treatments from any passages were shown a typical large and flat morphology as senescence cells.

Although there was no significantly difference of SA- $\beta$-gal positive cells in old stages by $40 \%$ and $60 \%$ GR treatments onset after $30 \mathrm{PD}$, much more cells in aging stages were kept slim as fibrous in morphology by $40 \%$ and $60 \%$ GR versus NG or HG, respectively (Fig. 4B, $\mathrm{C}$ and $\mathrm{D}$ ). These results indicate a potential cellular metabolic adaptive process under GR culture. Taken together, our results suggested that GR impacts on survival days or lifespan through suppressing cellular aging processes to lead a prolongation of lifespan in vitro.

The senescent cells with $\geq 95 \%$ SA- $\beta$-gal positive stain in HG medium were ahead of 8,8 , 7, 6 and 4 PD when started from 26, 30, 35, 40 and 45 PD versus NG groups, respectively (Fig. $4 \mathrm{~A}, \mathrm{~B}, \mathrm{C}, \mathrm{D}$ and $\mathrm{E}$ ). These results further conformed that HG culture induced cell senescence ahead of the normal control to develop into the premature aging as described above.

\section{Discussions}

CR impacts on aging from many animal studies have made it an attractive approach to potentially manipulate aging in humans. Although the effect of CR in animal models is obvious, some studies have revealed that the effects of CR-inducing longevity in experimental animal models have varied, which may be due to genetic variation between different species as well as different living conditions for experimental animals. Therefore, these uncontrolled factors in CR animal models may reduce its utility in mechanisms studies and the molecular mechanism of CR anti-aging remains not clear so for. It is important to evaluate more closely what the likely impacts of CR will be in human. Studies in rodents have been done at various levels of restriction below that of "control" fed animals [2, 19, 20]. Such results show that there is a strong linear positive relation between the degree of caloric restriction and the longevity benefit $[2,20]$. What level of CR could get the maximum benefits of anti-aging impacts in human? Animal study couldn't give the last answer. In order to provide directly evidences and the more precise molecular mechanisms of extending lifespan of CR for human, this paper was to elucidate the detail effects of glucose restriction (GR) on cell replicative senescence in vitro by human diploid fibroblasts IMR-90.

Using a CR mimic human cell model, our data show that survival days and lifespan of IMR-90 were extended under the situations of GR (Fig. 1A and B). The result suggests that the benefits of GR on longevity are related to the extent of glucose restriction. In other words, the higher level of GR, the greater relative benefit on extending survival day of IMR-90 could be acquired. The positive liner relationship was exited between GR levels and survival days increased relative to control (Fig. 1C), similar as a liner relation summered from CR animals between the degree of CR and the longevity benefit [2]. This result is in accord with CR animal observations that show an increase in lifespan is positively correlated to the degree of CR $[2,19,20]$. This study provides evidence that a GR culture cellular system may mimic CR effects in animals. And it's further established that CR can induce longevity not only in experimental animal models, but also in a culture cellular system $[11,13]$.

In contrast with GR impacts and control groups, HG treatment could significantly induce premature senescence of IMR-90 started from any passages. The impacts of HG on inducing aging become lager when HG was onset from older cells. This seems that older cells couldn't endure HG treatment and are more easily to be induced premature senescence than younger cells. It indicates that old cells of IMR-90 were more sensitive to HG impact 
on inducing premature senescence than young cells. This result is consistent with recent finding that high glucose concentration could induce premature senescence for 3 different types of normal human diploid fibroblasts (including IMR-90) [11].

Almost all CR animal studies have been initiated very early (often immediately postweaning) and maintained throughout life. It is completely unfeasible for human interventions to engage CR for their entire lives. What impacts on lifespan is a key question when CR is initiated in adult life. Relatively few studies are available in animals to answer this question [16-18]. However, human CR study is not feasible to achieve these results. In order to give the detail impacts of GR onset, we assessed GR effect on survival days of IMR-90 initiated from different passages. From the data shown in Fig. 2A and B, mean survival days were increased significantly whenever IMR-90 is initiated GR culture. But the extents of survival days increased were declined as initial passages addition. These data mean that it's never too late to start GR culture and the relative benefit of GR could be declined as initial passages addition. It indicates that the later GR onset, the smaller benefit could be acquired on IMR-90 longevity. These results are also consistent with CR animal studies that the CR effects have the same decline patterns as the age of onset of CR treatment $[2,16-18]$.

One important feature of GR revealed by our model is that the impacts of GR levels are larger than initial passages on retarding replicative senescence of IMR-90, which can be seen from Table 3 and Fig 2D. This agrees with empirical observations. We found that higher GR level onset from stable growth could have greater effect on extending survival days than lower GR level onset from the same stages. And even onset from old cells, higher GR level has the same effect on extending survival days as lower GR level onset from stable growth. This result told us that the higher GR level, the larger relative benefit on extending survival day could be acquired when IMR-90 was initiated from the same stages. This means that the GR levels have greater effect than initial passages on IMR-90 longevity.

The CPR of IMR-90 could be slow down rapidly by higher degree of GR (Fig. 3). In order words, it needs shorter time for higher level of GR to have effect on slowing CPR than lower level of GR. This indicates that higher level of GR could rapidly affect the CPR of IMR-90 than lower level of GR. This might be the reason why different GR levels and initial passages were associated with the impact on extending survival days of IMR-90. These results further supported that GR retarding replicative senescence of IMR-90 was associated with not only the extent of GR degree but also initial passages and the impact of GR degree was larger than initial passages.

In our previous study, we have observed some genes expression of senescence and cell cycle such as p16, p53 and p21 to find the possible molecular mechanisms of GR effects [21]. We found that lower expression of p16 and p21 might be associated with GR effects on retarding replicative senescence of IMR-90 and this result was consistent with Y Li's conclusion that decreased expression of $\mathrm{p} 16$ was induced by GR [11]. Epigenetic mechanisms have recently been recognized as major contributors to nutrition-related longevity and aging control $[11,22]$. Two primary epigenetic codes, DNA methylation and histone modification, are believed to dynamically influence chromatin structure, resulting in expression changes of relevant genes. Genetic mechanisms on GR-induced longevity could be investigated by using this cellular model system for further studying CR anti-aging approaches in vitro.

Taken together, GR impacts on IMR-90 longevity were related not only with GR degrees but also initial passages. The relative benefits of GR on extending survival days of IMR-90 were declined with initial passages addition. But the impacts of GR degree on retarding replicative senescence was greater than onset time of IMR-90.The pattern of declining benefit of GR with lower GR degree and initial passages addition is entirely consistent with the results from CR animals that the benefits of CR on lifespan extension are declined with lower extent of caloric restriction and late onset [2]. These conclusions further established that CR can induce longevity not only in experimental animal models, but more importantly, in a culture cellular system, which may provide an excellent in vitro model for CR studies. We hope to investigate the possible molecular mechanism of CR anti-aging by using this cellular CR model in further study. 
Jin/Zhang: Effects of Glucose Restriction on Retarding Replicative Senescence

\section{Acknowledgements}

This study was supported by grant from National Department Public Benefit Research Foundation by Ministry of Health P. R. China (No.201302008).

\section{References}

1 McCay CM, Crowell MF, Maynard LA: The effect of retarded growth upon the length of life span and upon the ultimate body size. J Nutr 1935;10:63-79.

-2 Speakman JR,Hambly C: Starving for Life: What animal studies can and cannot tell us about the use of caloric restriction to prolong human lifespan. J Nutr 2007;137:1078-1086.

-3 Fontana L, Partridge L, Longo VD: Extending healthy life span from yeast to humans. Science 2010;328:321-326.

-4 Colman RJ, Anderson RM, Johnson SC, Kastman EK, Kosmatka KJ, Beasley TM, Allison DB, Cruzen C, Simmons HA, Kemnitz JW, Weindruch R: Caloric restriction delays disease onset and mortality in rhesus monkeys. Science 2009;325:201-204.

5 Cruzen C, Colman RJ: Effects of caloric restriction on cardiovascular aging in non-human primates and humans. Clin Geriatr Med 2009;25:733-743.

6 Holloszy JO, Fontana L: Caloric restriction in humans. Exp Gerontol 2007;42:709-712.

7 Roth GS, Lane MA, Ingram DK, Mattison JA, Elahi D, Tobin JD, Muller D, Metter EJ: Biomarkers of caloric restriction may predict longevity in humans. Science 2002;297:811.

-8 Pendergrass WR, Li Y, Jiang D, Fei RG, Wolf NS: Caloric restriction: conservation of cellular replicative capacity in vitro accompanies life-span extension in mice. Exp Cell Res 1995;217:309-16.

-9 Walford R, Weber L, Panov S: Caloric restriction and aging as viewed from biosphere 2. Receptor 1995;5:29-33.

10 Das SK, Gilhooly CH, Golden JK, Pittas AG, Fuss PJ, Cheatham RA, Tyler S, Tsay M, McCrory MA, Lichtenstein AH, Dallal GE, Dutta C, Bhapkar MV, Delany J P, Saltzman E, and Roberts SB: Long-term effects of 2 energyrestricted diets differing in glycemic load on dietary adherence, body composition, and metabolism in CALERIE: a 1-y randomized controlled trial. Am J Clin Nutr 2007;85:1023-1030.

$\checkmark 11$ Li Y, Tollefsbol TO: p16INK4a suppression by glucose restriction contributes to human cellular lifespan extension through SIRT1-mediated epigenetic and genetic mechanisms. PLoS ONE 2011;6:e17421.

12 Killilea DW, Ames BN: Magnesium deficiency accelerates cellular senescence in cultured human fibroblasts. Proc Natl Acad Sci USA 2008;105:5768-5773.

13 Jin J, Zhang T, Zhang E, Han C, Wu X, Xu X, Zhang Z, Tong T: A cell model mimic caloric restriction in vitro with human diploid fibroblast IMR-90 was constructed to retard replicative senescence. Chin J Biochem Mol Bio 2004;20:545-550.

14 Hayflick L: The limited in vitro lifetime of human diploid cell strains. Exp Cell Res 1965;37:614-636.

15 Nichols WW, Murphy DG, Cristofalo VJ, Toji LH, Greene AE, Dwight SA: Characterization of a new human diploid cell strain, IMR-90. Science 1977;196:60-63.

16 Weindruch R, Walford RL: Dietary restriction in mice beginning at 1 year of age: effect on life-span and spontaneous cancer incidence. Science 1982;215:1415-8.

17 Lipman RD, Bronson RT, Wu D, Smith DE, Prior R, Cao G, Han SN, Martin KR, Meydani SN, Meydani M: Disease incidence and longevity are unaltered by dietary antioxidant supplementation initiated during middle age in C57BL/6 mice. Mech Ageing Dev 1998;103:269-84.

18 Spindler SR: Rapid and reversible induction of the longevity, anticancer and genomic effects of caloric restriction. Mech Ageing Dev 2005;126:960-966.

19 Weindruch R, Walford RL, Fligiel S, Guthrie D: The retardation of aging in mice by dietary restriction: longevity, cancer, immunity and lifetime energy intake. J Nutr 1986; 116(4):641-54.

20 Hou C, Bolt K, Bergman A: A general life history theory for effects of caloric restriction on health maintenance. BMC Syst Biol 2011;5:78.

21 Jin J, Zhang T, Han C, Zhang E, Zhang Y, Zhang Z, Tong T: Effect of caloric restriction on expressions of senescence-associated genes of IMR-90 cells. Chin J Geriatr 2007;26:520-523.

-22 Li Y, Daniel M, Tollefsbol TO: Epigenetic regulation of caloric restriction in aging. BMC Medicine 2011;9:98. 